\title{
Experimental Endoscopic Cordotomy in the Sheep Model
}

\author{
Ali DALGIC ${ }^{1}$, Murat CALISKAN², Pinar CAN², Fatih ALAGOZ¹, Yahya GUVENC ${ }^{1}$, Ayhan OCAKCIOGLU1, \\ Oktay ALGIN ${ }^{3}$, Ergun DAGLIOGLU', Esra ERDEN', Omer BESALTI², Deniz BELEN ${ }^{1}$ \\ ${ }^{1}$ Ankara Numune Educational and Training Hospital, Neurosurgery Clinic, Ankara, Turkey \\ ${ }^{2}$ Ankara University, School of Veterinary Medicine, Department of Surgery, Ankara, Turkey \\ ${ }^{3}$ Ataturk Research and Training Hospital, Radiology Clinic, Ankara, Turkey \\ ${ }^{4}$ Ankara University, School of Medicine, Department of Pathology, Ankara, Turkey
}

\section{ABSTRACT}

AIM: Interventional pain therapies are usually based on destruction of the related pain-conducting pathways. Current procedures targeting pain have replaced conventional pain treatment modalities while being less invasive. In this study, we investigated the feasibility of the endoscopic percutaneous cordotomy process on the sheep cervical spinal cord.

MATERIAL and METHODS: Seven male sheep, Akkaraman ${ }^{\circledR}$ genus, were operated on in the study. The guide was introduced at $\mathrm{C} 1$ to $\mathrm{C} 2$ vertebrae. The interlaminar area was exposed by a dilator, the dura was identified, and then the working cannula was inserted into the subarachnoid space. The target point of cordotomy was defined by endoscopic visualization as the midpoint between the dentate ligament and ventral root entry zone. After determination of the target point, a carbon dioxide laser (CDL) probe was introduced through the cannula. Ablative lesioning was performed by CDL. Hindlimb withdrawal thresholds were measured using the "Sample Pain Scale". The lesion was demonstrated by magnetic resonance imaging and histopathological examination.

RESULTS: Three sheep had ipsilateral hemiparesis and the response to firm pressure test was not performed on them. Among the remaining four sheep, the pain tolerance test showed that one sheep was at stage 0 , two at stage 1 , and the last one at stage 3 .

CONCLUSION: Cordotomy might be successfully performed with the endoscopic technique in the sheep model and this should encourage future studies regarding minimal invasive procedures for intractable pain.

KEYWORDS: Cordotomy, Percutaneous technique, Endoscopy, Sheep

\section{INTRODUCTION}

Interventional therapies for pain in neurosurgery are usually based on destruction of the related pain-conducting pathways. Cordotomy, myelotomy, sympathectomy, DREZotomy, rhizotomy, and ganglionectomy are primary procedures utilized in selected intractable pain patients $(2,3,9,13)$. The vast majority of pain in the cancer population is nociceptive, which generally is more responsive to opioids and more amenable to destructive procedures $(1,2,8,9,12)$.

Current procedures targeting pain have replaced conventional pain treatment modalities while being less invasive and safer.
Conventional open cordotomies include a posterior midline cervical or thoracic approach that necessitates removal of lamina and a dural incision (2). Cervical cordotomies have recently been performed under indirect visualization of the spinal cord using fluoroscopic guidance and intrathecal contrast material injection $(1,8,9,12)$. Percutaneous cervical cordotomy (PCC) is the most useful procedure for patients with unilateral intractable pain, cancer and benign spinal disorders $(2,12,13)$. Computed tomography guided PCC, as described by Kanpolat et al. $(8,9)$, is a selective, safe and effective procedure with a lower morbidity and mortality rate. 
Minimally invasive procedures have improved substantially in the last two decades and the practice of endoscopic interventions such as adhesiolysis, discectomy or epiduroscopy have increased tremendously (5). More recently, intradural lesions have been treated by endoscopic methods such as arachnoid cyst fenestration and filum terminale release $(4,11)$. So far, there is no experimental or neuroanatomical study that points to endoscopic approaches for cordotomy. In the present study, we investigated the feasibility of the endoscopic percutaneous cordotomy process on the sheep cervical spinal cord.

\section{MATERIAL and METHODS}

Seven male sheep, Akkaraman ${ }$ genus, weighing between 20 and $23 \mathrm{~kg}$ and aged 3 years old were used for the study. All experimental procedures were approved by the Ethical Committee for Animal Care and Use at the Veterinary Medicine Faculty of Ankara University The protocol of the study was prepared in accordance with the guidelines of National Institutes of Health and of the International Association for the study of Pain (IASP).

\section{Anesthesia}

The procedure was performed after 24 hours of fasting. The preanesthetic preparation included intramuscular (IM) application of ketamine at a dose of $600 \mathrm{mg}$ and buprenorphine at a dose of $0.3 \mathrm{mg}$. The external jugular vein was cannulated and $200 \mathrm{mg}$ ketamine was injected intravenously. A single vial of cefazolin was also injected intravenously at a dose of $1 \mathrm{gram}$ as a prophylactic antibiotic. After intubation, the sheep were maintained with isoflurane (2\%) anesthesia and mechanically ventilated. The sheep received intravenous fluids $(0.9 \%$ normal saline). Body temperature was maintained with a circulating water pad. Oxygen saturation was monitored and maintained between $98 \%$ and $100 \%$. The heart rate was maintained within 80 to 120 beats per minute. Systolic pressure was maintained between 80 and $120 \mathrm{mmHg}$ and diastolic pressure was within 60 and $80 \mathrm{mmHg}$.

\section{Surgical Procedure}

Sheep were positioned prone with the hindlimbs cephalad beside the abdomen. The cervical area was prepared with povidone iodine. A small incision necessary for the introduction of endoscope to the surgical field was marked. A far-lateral vertical cut was made over the $\mathrm{C} 1$ to $\mathrm{C} 2$ vertebrae. The interlaminar area was exposed by a dilator and the dura was identified at the intersection of the facet joint and lamina. A catheter was placed into the subarachnoid space and cerebrospinal fluid (CSF) flow through the catheter confirmed the passage.

The working cannula was inserted into the subarachnoid space and stayed relatively fixed by the skin. The cannula involves three separate holes for the endoscope, laser probe and irrigation. The endoscope $(0.9-\mathrm{mm}$ thickness, Mylotec Inc., USA) was placed by means of a cannula and provided a direct view of the spinal canal. It was possible to have a 70 degree field of clear vision through CSF at a $\times 40$ magnification and 0 degree angle of view. The pial surface of the spinal cord and its blood vessels, the arachnoid membrane, the dentate ligament, the dorsal and ventral root entry zone, and respective radicular blood vessels could be identified with endoscopic visualization. The target point of cordotomy was defined as the midpoint between the dentate ligament and the ventral root entry zone. After determination of the target point, a carbon dioxide laser (CDL) probe (Revolix duo dual mode laser (2 micron, solid state, cw laser system, Lisa laser products)) was introduced through second hole of the cannula while visual control was provided by the endoscope. The red light at the tip of the laser probe could easily be noticed with the endoscope and thus gentle advancement of the probe over the surface of the spinal cord or the exact point for cordotomy could also be easily recognized. Ablative lesioning was performed with the application of the CDL probe for 1 second. Just after lesioning, the probe was removed and the endoscope showed the lesion point over the spinal cord.

After completion of the experimental procedure, the sheep were inspected for the possibility of infection and motor function tests were performed to assess passive and active motions of the limbs. Hindlimb withdrawal thresholds were measured by the "Sample Pain Scale" (Table I). This scale was separated into three steps and the last section was used for scoring in the present experiment (response to firm pressure). A painful pinch at the tip of the limb was performed to assess limb withdrawal and the pressure was gradually increased if there was no movement over the hindlimb. The pressure prior to the loss of contact between the tip and the hindlimb was displayed and recorded on the meter. Three sheep were sacrificed for histopathological examination at the second week after surgery, and the remaining four sheep were studied for magnetic resonance imaging (MRI) scanning at the same time. The lesion was also demonstrated on MRI (Siemens $\AA$ Trio, Germany). To confirm the exact localization on MRI, sheep were scanned in the lateral decubitus position using a birdcage multi-channel head-cervical array coil and parallel imaging techniques. After acquisition of scout images, sagittal plane three-dimensional (3D) T1 weighted (W), 3D-T2W (SPACE sequence with variant flip-angle mode) and twodimensional (2D) T2W gradient-echo images were obtained. Total MRI time was approximately 30 minutes for each sheep. The data regarding the details of MRI protocols are presented in Table II. All MRI examinations were evaluated by the same radiologist.

\section{RESULTS}

There was no complication of the procedure or mortality but three sheep had ipsilateral hemiparesis (mild in one and moderate in two). Response to the firm pressure test was not performed for these three sheep. These sheep were euthanized and the upper cervical spinal cord was removed for histopathological examination to evaluate microscopic changes at the lesion site (the remaining four sheep were reserved for MRI studies). On histopathological sections, microcystic degeneration, lysis and cellular loss were the prominent findings at the lesion site. An inflammatory reaction rich in macrophages and cellular loss were noted in the neighboring spinal cord sections (Figure 1A, B). 
Table I: Sample Pain Scale

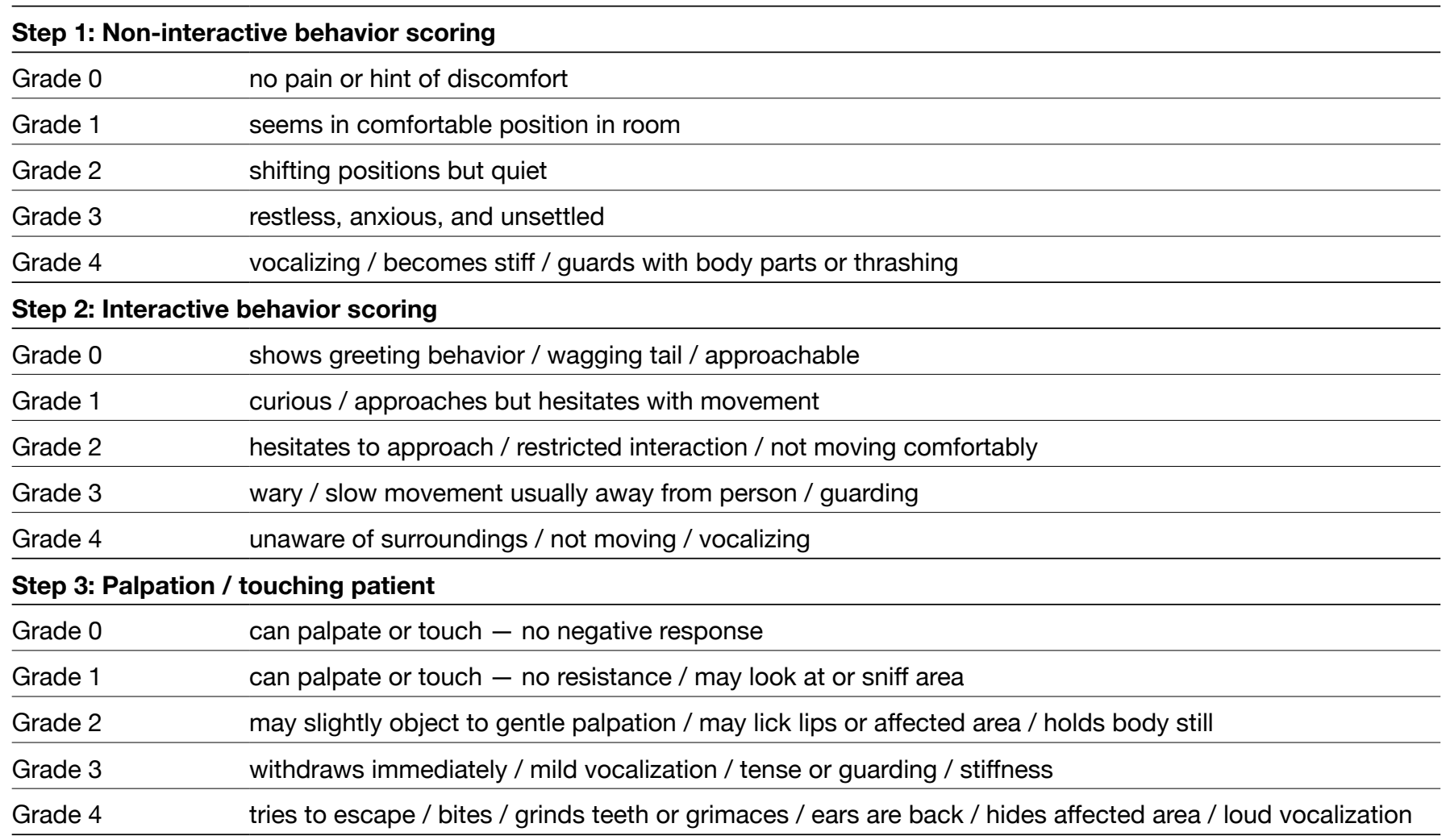

Table II: The Data Regarding the Details of MRI Protocols

\begin{tabular}{lcc}
\hline & 3D-MPRAGE & 3D-SPACE \\
\hline TR/TE $(\mathrm{ms})$ & $2130 / 3.45$ & $3000 / 579$ \\
\hline TI (ms) & 1100 & - \\
\hline Slice thickness & $0.8 \mathrm{~mm}$ & $0.6 \mathrm{~mm}$ \\
\hline FOV $^{*}(\mathrm{~mm})$ & $230 \times 230$ & $240 \times 240$ \\
\hline Acquisition time & $5.5 \mathrm{~min}$. & $6 \mathrm{~min}$. \\
\hline NEX & 1 & 2 \\
\hline Number of slices & 240 & 240 \\
\hline Flip angle & $8^{\circ}$ & Variable \\
\hline Imaging plane & Sagittal & Sagittal \\
\hline Distance factor & $50 \%$ & - \\
\hline PAT factor & 2 & 2 \\
\hline PAT mode & GRAPPA & GRAPPA \\
\hline Voxel size (mm) & $0.8 \times 0.8 \times 0.8$ & $0.6 \times 0,6 \times 0,6$ \\
\hline FA mode & - & T2 variant \\
\hline Abbreviations: & & \\
\hline
\end{tabular}

Abbreviations: TI: Time of inversion, 3D-SPACE: Three-dimensional sampling perfection with application-optimized contrasts using different flip angle evolutions, 3D-MPRAGE: Three-dimensional T1W magnetization prepared rapid acquisition gradient-echo, NEX: Number of excitations, FOV: Field of view, PAT: Parallel acquisition technique, GRAPPA: Generalized auto calibrating partially parallel acquisitions.
The remaining four sheep for MRI were also evaluated with the "Multifactorial Pain Scale". The pain tolerance test showed that one sheep was at stage 0 , two at stage 1 and the last one at stage 3 (no change in pain scale). MRI confirmed a spinothalamic pathway lesion at the $\mathrm{C} 1$ level of the cervical spinal cord (Figure 2A, B).

\section{DISCUSSION}

Cordotomy is an alternative treatment option to medical treatment alone in cancer patients with intractable pain. In the present study, we investigated the endoscopic feasibility of interventional pain procedures in a sheep model.

Patient selection for neurosurgical pain procedures should be based on pain characteristics and so far treatment has been essentially more useful for nociceptive intractable pain (2, 9, $12,13)$. The best candidates for cordotomy are patients with unilateral somatic cancer pain and compression of the plexus, roots, or nerves $(1,8,12)$. Pain is one of the major complaints of cancer patients and approximately $75 \%$ of advanced stage cancer patients experience pain (8).

The World Health Organization (WHO) analgesic protocol can provide adequate pain relief in over $90 \%$ of adults with cancer. However, there remains a small minority where this protocol is ineffective because of incomplete response or adverse effects limiting dose escalation of systemic analgesics. Up to $90 \%$ of this minority group may benefit from interventional pain management techniques (3). Opioids, whether given in 


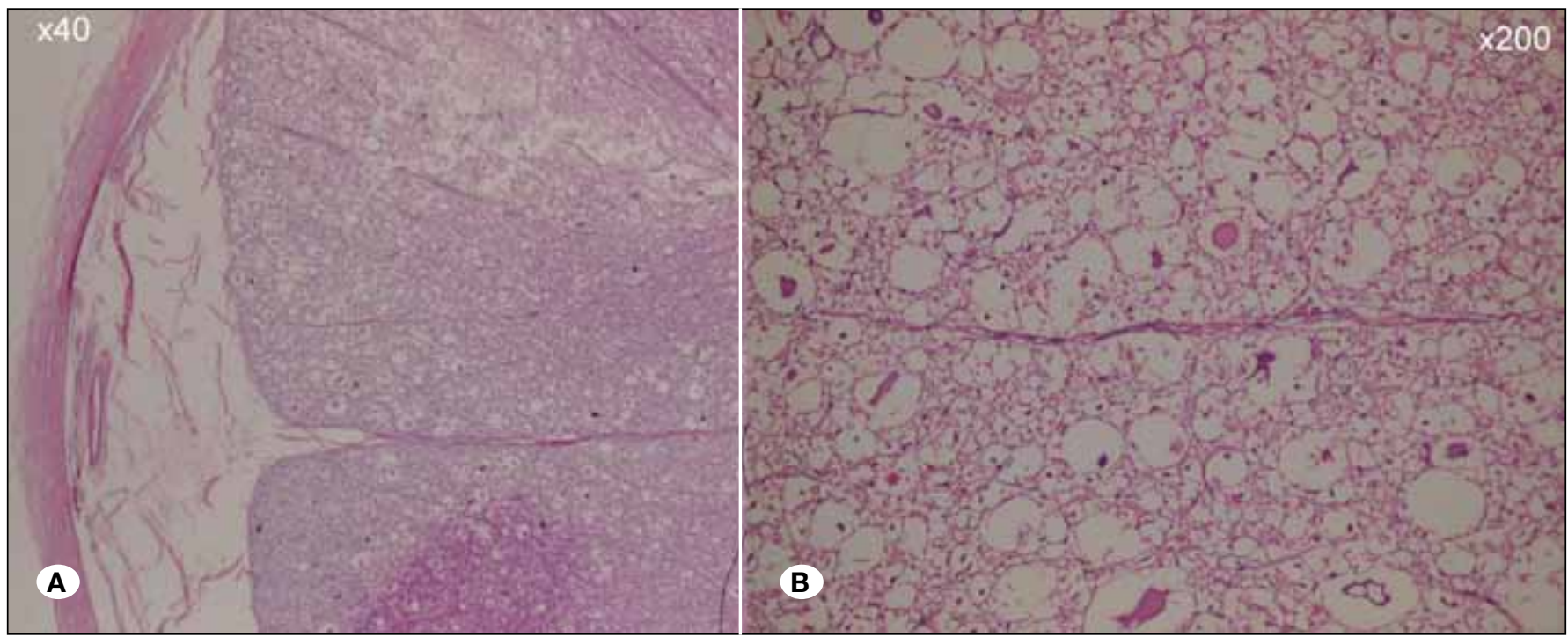

Figure 1: The findings at the laser lesion of the spinothalamic pathway such as microcystic degeneration, lysis and cellular loss are shown.
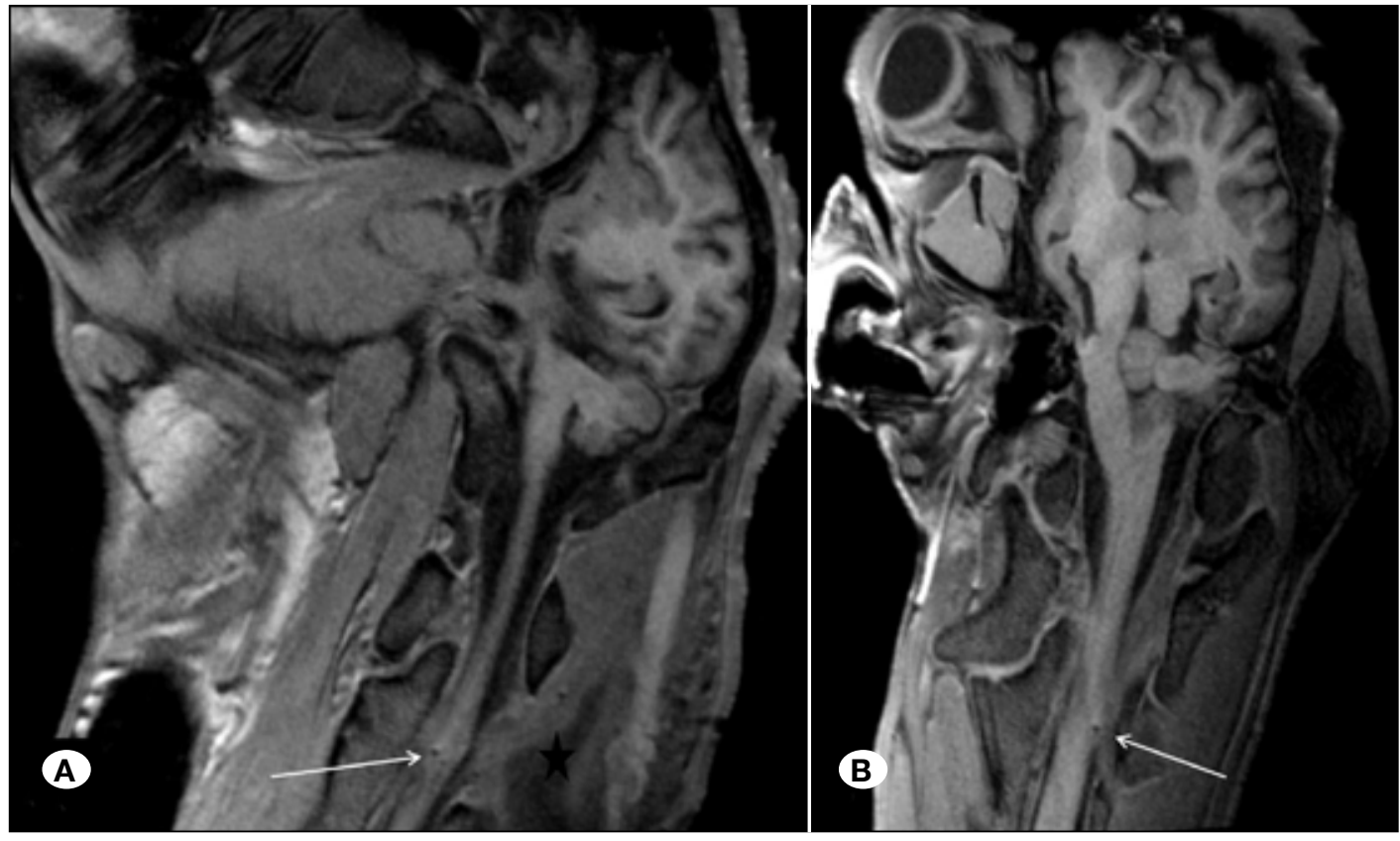

Figure 2: Magnetic Resonance Imaging reveals the lesion at the spinothalamic pathway lesion at the $\mathrm{C} 1$ level (white arrow).

intravenous preparations or in central nervous system (CNS) opioid pumps, are indicated and beneficial for a considerable number of cancer patients with intractable pain. However, this measure is unsatisfactory in a minority of cases and this group might be considered for pain surgery as a therapeutic option. Ablation of the lateral spinothalamic tract in the anterolateral segment of the spinal cord is effective for the treatment of intractable cancer pain $(1,3,8,9,12,13)$.

Physicians involved in interventions for ablative pain procedures reserve destructive procedures for terminal stage cancer patients but destructive procedures like cordotomy might readily be an earlier alternative. It is obvious that the quality of life for these patients will be increased and they will return to an active life once there is marked denervation at the painful area. Cordotomy and other destructive pain procedures with computed tomographic guidance can be successfully performed in selected patients with a high success rate $(8,9)$. Kanpolat et al. reported a $92.5 \%$ success rate with percutaneous cordotomy and a relatively lower rate of complications. Cordotomy may be considered as a treatment option to control unilaterally localized chronic cancer pain states (9).

Cordotomy procedures have been performed by radiofrequency (RF) lesioning so far. Recently, a new version of RF electrode that can perform neurophysiological tests such as 
impedance measurement and stimulation was introduced to the field. This provides exact localization of the target point. However, RF probes are rigid and inadequate for flexible endoscope systems. Fonoff et al. presented a case report with endoscopic-guided percutaneous RF cordotomy (7). They removed the endoscope from the field after complete visualization of the target zone for a complete RF cordotomy. The cannula was kept in place and the RF probe was introduced into the surgical field. After completion of the procedure, the endoscope was re-inserted again for visualization of the subarachnoid space (7). We used a flexible laser probe that provided real-time visual control and was safe to explore the target point. On the other hand, the working cannula included two channels for the endoscope and probe, so both the endoscopic viewing and the manipulations could be synchronously done. The cannula needs to have a fine and flexible bending angle and should allow fine manipulations within the dura or around the spinal cord. This feature increases applicability and improves the visual ability of the flexible laser probe.

In the past, laser lesioning was used as a primary treatment procedure $(6,10,14)$. Fink treated a case who suffered from intractable rectal pain with commissural myelotomy using CDL (6). Subsequently, CDL was used for the dorsal root entry zone (DREZ) procedure with an acceptable success rate $(10,14)$. The RF lesioning technique provides control of the electrode temperature and the procedure can be carried out in a safer manner. However, these two techniques have similar success rates (14). Laser lesioning has several advantages over the DREZ procedure. Laser lesions are definitely easier to create and may be created rapidly due to the brief duration of each lesion (10). Although the lesion is localized relatively superficially with mild penetration into the surrounding spinal cord, there is usually a disparity between the laser beam size and true laser beam size that is compatible with the finding of unexpectedly large lesions (10). On the other hand, Young et al. reported several problems about laser lesions such as movement of the spinal cord during inspiration-expiration leading to misdirected lesions, accidental injury to blood vessels, or hazardous effects on neural tracts (14).

The popularity of destructive surgical pain procedures has diminished in clinical practice in the last decades. This has been attributed to the high efficacy of new methods such as morphine pumps or neurostimulation but there has been an overt neglect of functional procedures regarding pain procedures and electrode technology (9). Laser lesioning is commonly used for the DREZ operation but cordotomy was not performed with a laser probe until the present study. Although laser lesioning might cause lesions that are unexpectedly larger or smaller than the targeted lesion size, lesioning was managed completely with endoscopic flexible probe manipulation. We believe that the technology of flexible $\mathrm{RF}$ probes or the laser probe needs to be improved to create a more predictable lesion size.
In conclusion, cordotomy might be successfully performed with full endoscopic assistance in a sheep model and this should encourage future studies regarding minimal invasive functional procedures for a better control of intractable pain.

\section{REFERENCES}

1. Bekar A, Kocaeli H, Abas F, Bozkurt M: Bilateral high-level percutaneous cervical cordotomy in cancer pain due to lung cancer: A case report. Surg Neurol 67(5): 504-507, 2007

2. Cetas JS, Saedi T, Burchiel KJ: Destructive procedures for the treatment of nonmalignant pain: A structured literature review. J Neurosurg 109(3): 389-404, 2008

3. Crul BJ, Blok LM, van Egmond J, van Dongen RT: The present role of percutaneous cervical cordotomy for the treatment of cancer pain. J Headache Pain 6(1): 24-29,2005

4. Di X: Endoscopic spinal tethered cord release: Operative technique. Childs Nerv Syst 25(5): 577-581, 2009

5. Donato AD, Fontana C, Pinto R, Beltrutti D, Pinto G: The effectiveness of endoscopic epidurolysis in treatment of degenerative chronic low back pain: A prospective analysis and follow-up at 48 months. Acta Neurochir Suppl 108: 67-73, 2011

6. Fink RA: Neurosurgical treatment of nonmalignant intractable rectal pain: Microsurgical commissural myelotomy with the carbon dioxide laser. Neurosurgery 14(1): 64-65,1984

7. Fonoff ET, de Oliveira YS, Lopez WO, Alho EJ, Lara NA, Teixeira MJ: Endoscopic-guided percutaneous radiofrequency cordotomy. J Neurosurg 113(3): 524-527,2010

8. Kanpolat Y, Ozdemir M, Al-Beyati E: CT-guided percutaneous cordotomy for intractable pain in what is more than a disease: Lung malignancies. Turk Neurosurg 23(1): 81-87,2013

9. Kanpolat $\mathrm{Y}$, Ugur $\mathrm{HC}$, Ayten $\mathrm{M}$, Elhan $\mathrm{AH}$ : Computed tomography-guided percutaneous cordotomy for intractable pain in malignancy. Neurosurgery 64 Suppl 3:ons187-194, 2009

10. Levy WJ, Nutkiewicz A, Ditmore QM, Watts C: Laser-induced dorsal root entry zone lesions for pain control. Report of three cases. J Neurosurg 59(5): 884-886,1983

11. Mauer UM, Gottschalk A, Kunz U, Schulz C: Arachnoscopy: A special application of spinal intradural endoscopy. Neurosurg Focus 30(4): E7, 2011

12. Raslan AM, Cetas JS, McCartney S, Burchiel KJ: Destructive procedures for control of cancer pain: the case for cordotomy. J Neurosurg 114(1): 155-70; 2011

13. Whitworth LA, Feler CA: Application of spinal ablative techniques for the treatment of benign chronic painful conditions: history, methods, and outcomes. Spine 27(22): 2607-12; 2002

14. Young RF: Clinical experience with radiofrequency and laser DREZ lesions. J Neurosurg 72(5): 715-20; 1990 